\title{
Facile synthesis of hollow urchin-like gold nanoparticles and their catalytic activity
}

\author{
Wei Wang • Yujia Pang • Jian Yan • Guibao Wang • \\ Hui Suo $\cdot$ Chun Zhao $\cdot$ Shuangxi Xing
}

Published online: 2 June 2012

(C) The Author(s) 2012. This article is published with open access at Springerlink.com

\begin{abstract}
The galvanic replacement reaction between $\mathrm{Ag}$ nanoparticles (NPs) and $\mathrm{HAuCl}_{4}$ followed by addition of ascorbic acid led to the formation of AuNPs sharing both urchin-like and hollow structures. The AgNPs took as sacrificial templates to guide the hollow structure and the intermediates provided rough surface and active sites for the further deposition of AuNPs, which originated from the reduction of excess $\mathrm{HAuCl}_{4}$ by ascorbic acid. These unique structured AuNPs presented excellent optical properties and great advantages in catalysis applications.
\end{abstract}

Keywords Hollow structure $\cdot$ Urchin-like structure $\cdot$ Gold nanoparticles $\cdot$ Catalytic activity

\section{Introduction}

Nanostructured gold nanoparticles (AuNPs) have revealed great potential applications in many fields owing to their unique optical and catalytic properties [1-6]. Among them, urchin-like or branched gold particles with a rough surface are of great significance. The high surface area endows them excellent performance in catalysis, surface plasmon resonance (SPR) [7], surface-enhanced Raman spectra (SERS) [8], electronic devices, and biological applications [1, 2,

W. Wang $\cdot$ Y. Pang $\cdot$ J. Yan $\cdot$ S. Xing $(\bowtie)$

Institute of Colloid and Interface Chemistry, Faculty of Chemistry,

Northeast Normal University,

Changchun 130024, People's Republic of China

e-mail: xingsx737@nenu.edu.cn

W. Wang $\cdot$ G. Wang $\cdot$ H. Suo $\cdot$ C. Zhao

State Key Laboratory on Integrated Optoelectronics, College of Electronic Science and Engineering, Jilin University, Changchun 130012, People's Republic of China
9].In particular, owing to the existence of a large electromagnetic field enhancement at the tips of branched particles, a strong SERS activity can be detected with intensity over $10^{7}$ and a relatively high reproducibility [8]. In addition, the urchin-like structure leads to large SPR shift from 500 to $800 \mathrm{~nm}$ and tunable SPR enhancement light scattering and absorption, making them novel and highly effective contrast agents for in vivo cancer diagnosis and therapy [10]. Many strategies emerge for the construction of size and branchcontrolled urchin-like gold particles [11], mainly through seeding growth approach [12-14], where the preferential deposition of atoms on certain facets is dominant [12]. Besides, various molecules are adapted as stabilizer or capping agent to guide the formation of urchin-like structures, such as thiol-terminated molecules [15], cetyltrimethylammonium bromide [8], and sodium dodecyl sulfate [16].

On the other hand, the hollow metal nanostructures present many potential applications in catalysts, drug delivery, optical imaging, and nano-reactors [17, 18]. In the hollow nanosphere system, SPR peak locations shift over a region of more than $100 \mathrm{~nm}$ due to changes of shell thickness [19], rendering them great potential application in optical sensors [17]. As for the generation of NPs with hollow interiors, the sacrificial template approach is the most adaptable one [18-21]. Generally, AgNPs have always been taken as sacrificial templates and their reaction with $\mathrm{HAuCl}_{4}$ or other metal salts leads to the formation of metal NPs with voids [17, 22-28], in which the morphology of the AgNP seeds directly controls that of the resulting metal shells. These hollow-structured materials have been explored for biomedical and catalytic applications. An improved performance has already been achieved for their use as contrast enhancement agents for both optical coherence tomography and photoacoustic tomography $[22,26]$.

In this paper, we provided a facile method to prepare gold particles sharing both hollow interior and urchin-like shell. 
Such a unique nanostructure facilitates the access of species to the Au surface and improves their catalytic performance [29].

\section{Experimental}

\section{Chemicals}

Deionized water was used in all the experimental processes (18.0 $\left.\mathrm{M} \Omega \mathrm{cm}^{-1}\right)$. Hydrogen tetrachloroaurate (III) $(\mathrm{Au} \geq 47.8 \%$ on metals basis), silver nitrate, polyvinylpyrrolidone (PVP), sodium borohydride were bought from Sinopharm Chemical Reagent Co. Ltd., ascorbic acid, sodium citrate, and glucose were from Beijing Chemical Works and all these above chemicals were used as received. 4-Nitrophenol (4-NP, Shanghai Chemical Reagent Co. Ltd.) was purified before use.

Synthesis of silver seeds

Typically, $4.5 \mathrm{mg}$ of $\mathrm{AgNO}_{3}$ was dissolved in $25 \mathrm{~mL}$ of water and preheated at $70^{\circ} \mathrm{C}$ for $10 \mathrm{~min}$. After that, the temperature was increased to $110^{\circ} \mathrm{C}$ followed by addition of $1.5 \mathrm{~mL}$ of sodium citrate ( $1 \%$ in mass) to initiate the reduction of $\mathrm{AgNO}_{3}$. After $1.5 \mathrm{~h}$, the colloidal Ag solution was cooled to room temperature.

The AgNPs in (ethylene) glycol (EG) system were prepared based on the previous report [30]: $0.025 \mathrm{~g}$ of $\mathrm{AgNO}_{3}$ and $0.10 \mathrm{~g}$ of PVP were dissolved in $10 \mathrm{~mL}$ of EG at room temperature. After that, the solution was heated at $160^{\circ} \mathrm{C}$ in an oil bath for $3 \mathrm{~h}$ under stirring.

Synthesis of hollow urchin-like gold nanoparticles

Four milliliters of $\mathrm{Ag}$ colloid was centrifuged at 4,000 rpm for 5 min and the concentrated $\mathrm{Ag}$ deposites were dropped into $1 \mathrm{~mL}$ of $\mathrm{HAuCl}_{4}$ aqueous solution $(2.94 \mathrm{mM})$. Right after that, $1 \mathrm{~mL}$ of ascorbic acid $(10 \mathrm{mM})$ was added. The solution turned dark blue in a short period of time. Five minutes later, $400 \mu \mathrm{L}$ of PVP (50 mM in monomer concentration) was mixed with the product solution to avoid the possible aggregation in the further purification process. A control experiment was conducted with the same conditions in the absence of $\mathrm{Ag}$ seeds. The morphology of the products was investigated by a highresolution transmission electron microscopy (HRTEM, JEOL$2100 \mathrm{~F}$ ) operated at $200 \mathrm{kV}$ and scanning electron microscopy (SEM, XL-30 ESEM FEG). The optical spectra of the samples were recorded on a UV2400PC UV-vis spectrometer.

Catalytic reaction for degradation of 4-NP

Three hundred microliters of as-prepared solution was concentrated to a total of $10 \mu \mathrm{L}$ by centrifugation at $6,000 \mathrm{rpm}$ for 4 min. After removal of the supernatant, the isolated AuNPs were immediately added to a freshly prepared reaction mixture (3 mL) containing 4-NP $(0.15 \mathrm{mM})$ and $\mathrm{NaBH}_{4}(5.5 \mathrm{mM})$. The optical property of the reaction system was analyzed by using a UV-vis spectroscopy (UV-2550) at every 5-min interval.

Electrocatalytic oxidation of glucose

A piece of indium-tin-oxide (ITO)-coated glass $(1 \times 5 \mathrm{~cm})$ was firstly pre-cleaned with acetone and immersed into a solution of $\mathrm{H}_{2} \mathrm{O}_{2}: \mathrm{NH}_{3} \cdot \mathrm{H}_{2} \mathrm{O} / \mathrm{H}_{2} \mathrm{O}(1: 1: 5 v / v)$ for 30 min. After that, the ITO glass was rinsed with deionized water and dried at $40^{\circ} \mathrm{C}$.

The cleaned ITO glass was treated with aqueous 3aminopropyltriethoxysilane ( $0.25 \mathrm{wt} \%$ ) for $15 \mathrm{~min}$. After washing, the glass was immersed in a purified hollow urchin-like AuNPs solution for $30 \mathrm{~min}$, washed with water, and dried in air.

The electrocatalytic oxidation of glucose was performed on a $\mathrm{CHI} 852 \mathrm{C}$ electrochemical workstation $(\mathrm{CH}$ Instruments, Chenhua Co., Shanghai, China). A conventional three-electrode cell was utilized with a saturated $\mathrm{Ag} / \mathrm{AgCl}$ electrode as the reference electrode, a platinum plate as the counter electrode, and the Au-loaded ITO glass as the working electrode. A potential scan in the range of $-0.6-0.85 \mathrm{~V}$ with a scan rate of $50 \mathrm{mV} \mathrm{s}^{-1}$ was implemented to explore the electrochemical behavior of glucose in stirred $0.1 \mathrm{M}$ $\mathrm{NaOH}$ aqueous solutions containing $1.33 \mathrm{mM}$ glucose.

The estimation of the electrochemically active surface area of the hollow urchin-like and spherical gold electrodes were carried out using cyclic voltammetry $(\mathrm{CV})$ and the RandlesSevcik equation for a reversible redox couple, which at $25^{\circ} \mathrm{C}$ is [31]

$I_{\mathrm{p}}=\left(2.69 \times 10^{5}\right) n^{3 / 2} A D^{1 / 2} v^{1 / 2} C_{\infty}$

where $I_{\mathrm{p}}$ is the peak current (ampere), $n$ is the number of electrons transferred, $A$ is the electrode area (square centimeter), $D$ is the diffusion coefficient of the electroactive species (square centimeter per second), $\nu$ is the scan rate (volt per second), and $C_{\infty}$ is the bulk concentration of the same electroactive species (moles per cubic centimeter). Here, we used $5 \mathrm{mM} \mathrm{K}_{3} \mathrm{Fe}(\mathrm{CN})_{6}$ in $0.1 \mathrm{M} \mathrm{KCl}$ aqueous solution for this purpose, and $\mathrm{K}_{3} \mathrm{Fe}(\mathrm{CN})_{6}$ has a diffusion coefficient of $1.0 \times 10^{-5} \mathrm{~cm}^{2} \mathrm{~s}^{-1}$ at $25^{\circ} \mathrm{C}$. The scan rate kept at $50 \mathrm{mV} \mathrm{s}^{-1}$ in the measurement.

\section{Results and discussion}

Figure $1 \mathrm{a}, \mathrm{b}$ present a typical TEM image of the resulting AuNPs. All observed particles are urchin-like with hollow interiors. The average particle size is $104 \pm 11 \mathrm{~nm}$ and the interior size ranges from 23 to $45 \mathrm{~nm}$. The interior turns smaller compared to the AgNP seeds $\left(d_{\mathrm{av}}=65 \mathrm{~nm}\right.$, Fig. $\left.1 \mathrm{c}\right)$, which may originate from the balance between the diffusion of the $\mathrm{Ag}^{+}$ions (or $\mathrm{Ag}$ atoms) and the shrinkage of $\mathrm{Au}$ 
Fig. 1 a, b TEM images of hollow urchin-like AuNPs at low and high magnification; c TEM image of Ag seeds obtained with centrifugation speed of $4000 \mathrm{rpm}$ for $5 \mathrm{~min}$; d EDS of the hollow urchin-like AuNPs; e TEM image of spherical AuNPs obtained in the absence of Ag seeds; $\mathbf{f}$ SEM image of hollow urchin-like AuNPs

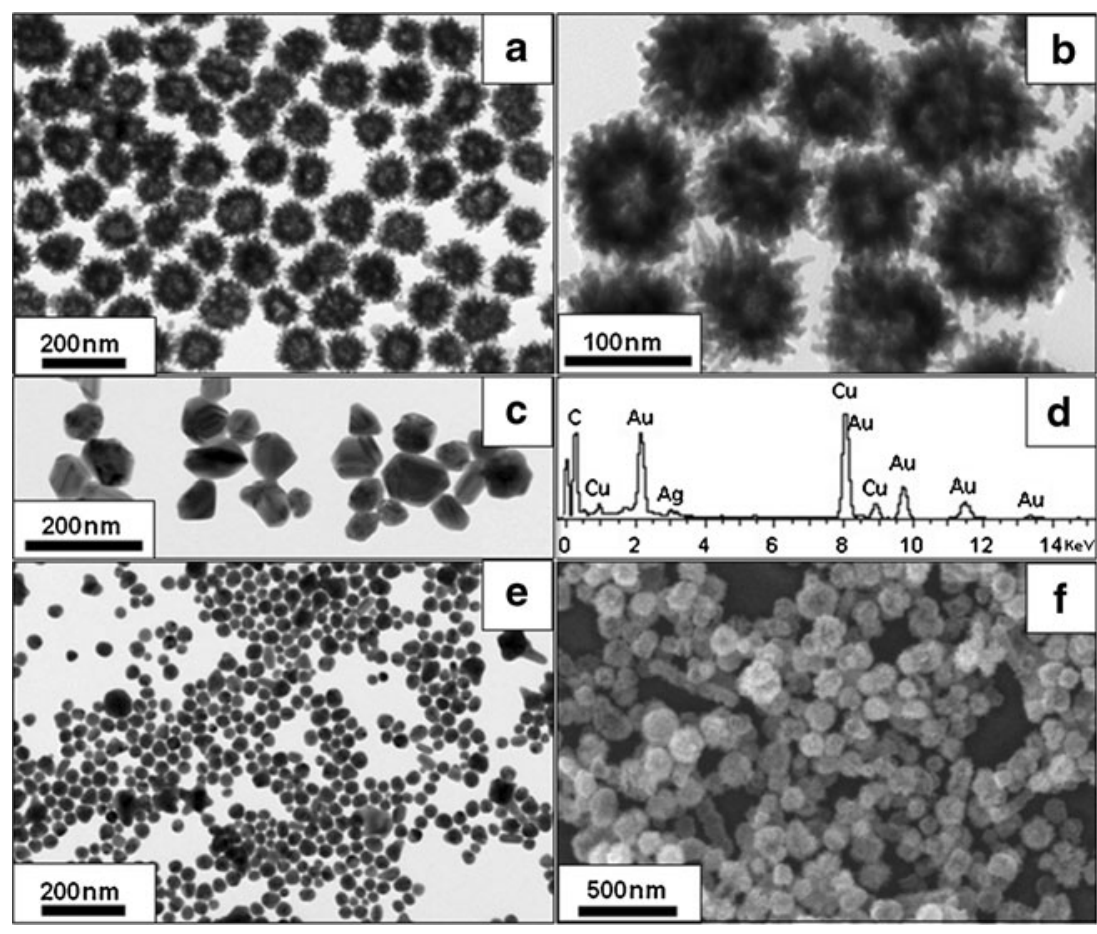

atoms. The galvanic replacement reaction between $\mathrm{Ag}$ and $\mathrm{HAuCl}_{4}$ leads to the dissolution of $\mathrm{Ag}$ atoms and their following migration to the outside [32]. Meanwhile, $\mathrm{HAuCl}_{4}$ is reduced to $\mathrm{Au}$ atoms and they deposit on the original Ag surface. Along with the disappearance of the AgNPs, AuNPs tend to shrink in order to minimize the surface energy. The diffusion rate of silver may be faster than that of the shrinkage of gold atoms; therefore, after the complete dissolution of Ag, the shrinkage process may stop, leaving the center part unoccupied. In comparison, only spherical AuNPs $\left(d_{\mathrm{av}}=33 \mathrm{~nm}\right)$ are obtained in the absence of Ag seeds (Fig. 1e). The SEM image of the products confirms that all the particles present rough surface with sharp tips (Fig. 1f).

The UV-vis spectra verify the structure evolution process (Fig. 2). Initially, the SPR peak of Ag colloid is observable at $420 \mathrm{~nm}$ (curve 1). Upon mixing the AgNPs with $\mathrm{HAuCl}_{4}$ solution, the original Ag peak red-shifts to $550 \mathrm{~nm}$ (curve 2). This indicates the beginning of the galvanic reaction between $\mathrm{Ag}$ seeds and $\mathrm{HAuCl}_{4}$ and partial formation of the nanoshells. After addition of ascorbic acid into the above system, the peak continues red-shifting to nearly $830 \mathrm{~nm}$ (curve 3), which derives from the combination of formation of the nanoshells and the growth of the Au branches onto their surface. In this sense, this structured NP can be found in application in optics and clinical diagnostics [22].

The ratio of $\mathrm{HAuCl}_{4}$ to $\mathrm{AgNP}$ seeds plays an important role in the formation of uniform hollow urchin-like AuNPs. Decreasing the amount of AgNP seeds by half results in the free growth of spherical AuNPs in bulk solution (Fig. 3a). Onto the AgNPs surface, only limited $\mathrm{HAuCl}_{4}$ is consumed to generate the aimed gold structures. The excessive $\mathrm{HAuCl}_{4}$ relative to the typical usage will be reduced into spherical AuNPs coexisting with the hollow ones. On the other hand, if we decrease the $\mathrm{HAuCl}_{4}$ content to half, less uniform structure is achieved (Fig. 3b), which should result from the incomplete dissolution of $\mathrm{Ag}$ or $\mathrm{AgCl}$ on the surface (vide post).

The selection of reductant appears significant for the generation of well-structured hollow urchin-like AuNPs. If $\mathrm{NaBH}_{4}$ was applied instead of ascorbic acid, no uniform urchin-like products were observed (Fig. 3c). Only small NPs with average diameter of $7 \mathrm{~nm}$ are distributed around

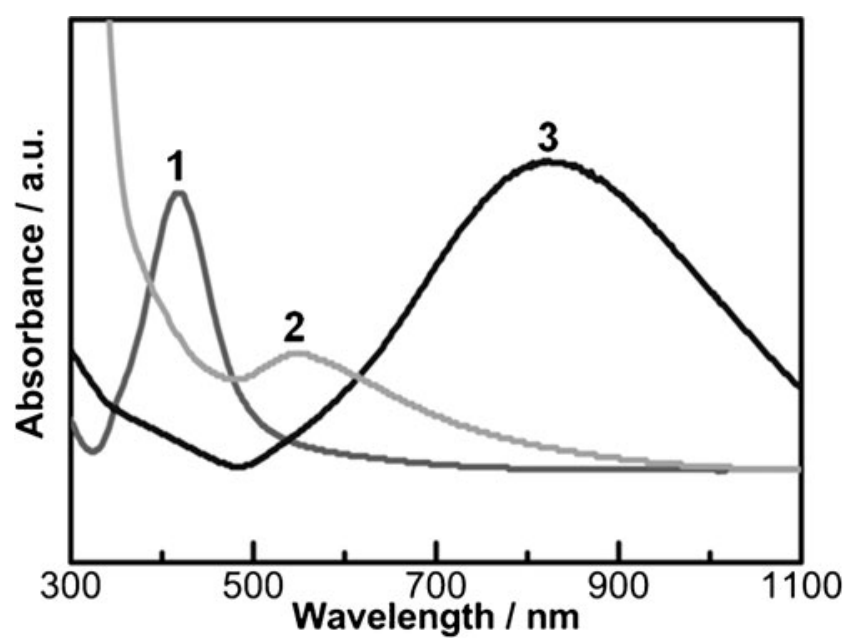

Fig. 2 UV-vis spectra of synthetic mixture. Curve 1: Ag colloid; curve 2: addition Ag seeds into $\mathrm{HAuCl}_{4}$ aqueous solution; curve 3: addition of ascorbic acid into the above solution 
Fig. 3 TEM images of AuNPs obtained from a system a with less Ag seeds; b with lower $\mathrm{HAuCl}_{4}$ concentration; $\mathbf{c}$ with $\mathrm{NaBH}_{4}$ as reductant instead of ascorbic acid; $\mathbf{d}$ operated at $100^{\circ} \mathrm{C}$

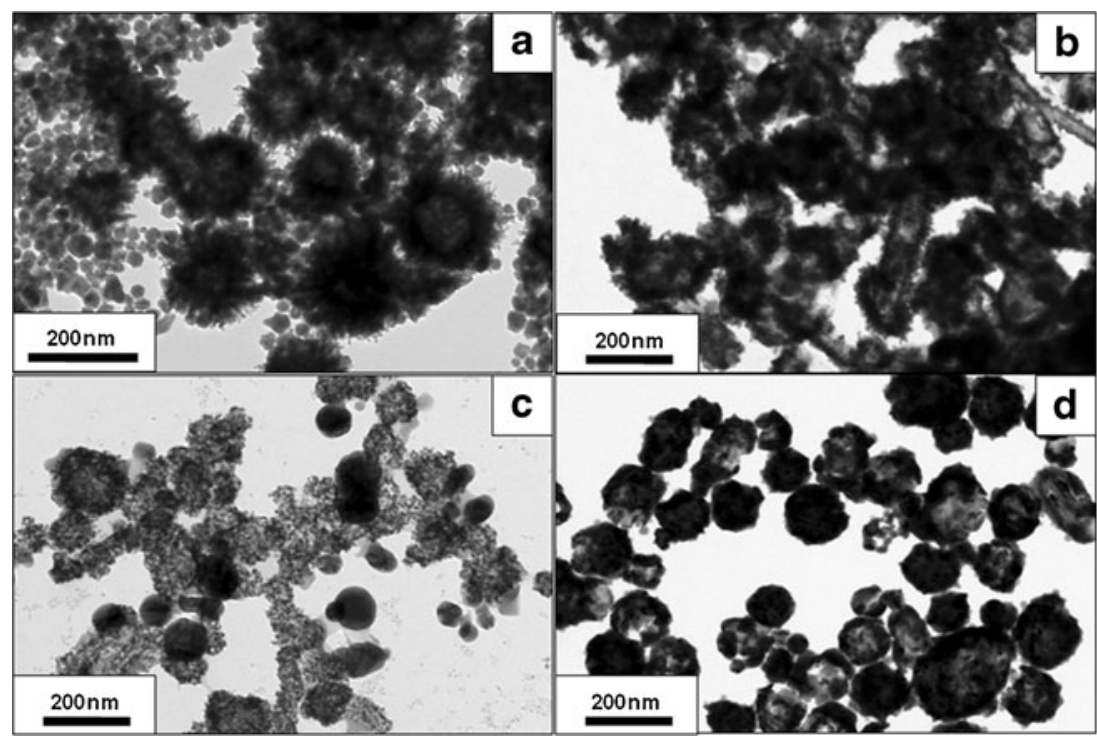

the as-obtained hollow gold structures. This indicates that a very fast reaction $\left(\mathrm{NaBH}_{4}\right.$ is a stronger reductant than ascorbic acid) does not benefit the further deposition of gold on the preexisting hollow gold surface.

The formation of the hollow urchin-like gold nanostructure can be induced by two separated steps (Fig. 4): (1) upon introducing concentrated $\mathrm{AgNPs}$ into the $\mathrm{HAuCl}_{4}$ aqueous solution, a galvanic replacement reaction occurs on the original AgNPs surface. After that, the Au atoms are generated and AgNPs simultaneously transfer into $\mathrm{Ag}^{+}$ions (or Ag atoms) followed by migration to the bulk solution (vide ante). Attention should be paid that no heat treatment is conducted, which allows for the fast diffusion of the newly formed $\mathrm{Au}$, forming alloy with the coexisting $\mathrm{Ag}$ atoms and constructing to seamless shell [24]. (2) Owing to the rough surface provided via the presence of undissolved $\mathrm{AgCl}$ [27], an urchin-like structure is readily produced when ascorbic acid is added to reduce the excessive $\mathrm{HAuCl}_{4}$. This occurs because the reduced gold has great tendency to nucleate on the surface of preexisting NPs for lowering the energy cost. In the case of $\mathrm{NaBH}_{4}$ system, a kinetic process may dominate in the second step. In addition, $\mathrm{AgCl}$ taking as archoring sites can be coordinated or dissolved via the excess addition of $\mathrm{HAuCl}_{4}$, and no $\mathrm{Cl}$ signal is detected in energy-dispersive spectrometer (EDS, Fig. 1d, the Cu peaks come from the TEM grid). Finally, such interesting structured AuNPs are achieved. A control experiment was done by carrying out the reaction at $100^{\circ} \mathrm{C}$. As mentioned in the previous reports, such condition helps for the generation of

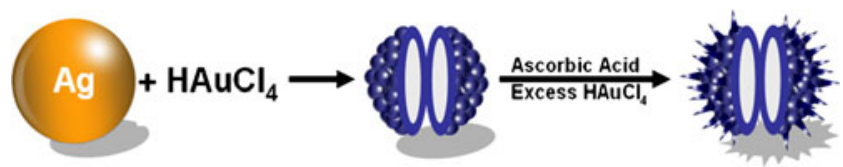

Fig. 4 Scheme for the fabrication of hollow urchin-like AuNPs seamless and smooth metal shell without the presence of $\mathrm{AgCl}$. As a result, introducing ascorbic acid does not lead to

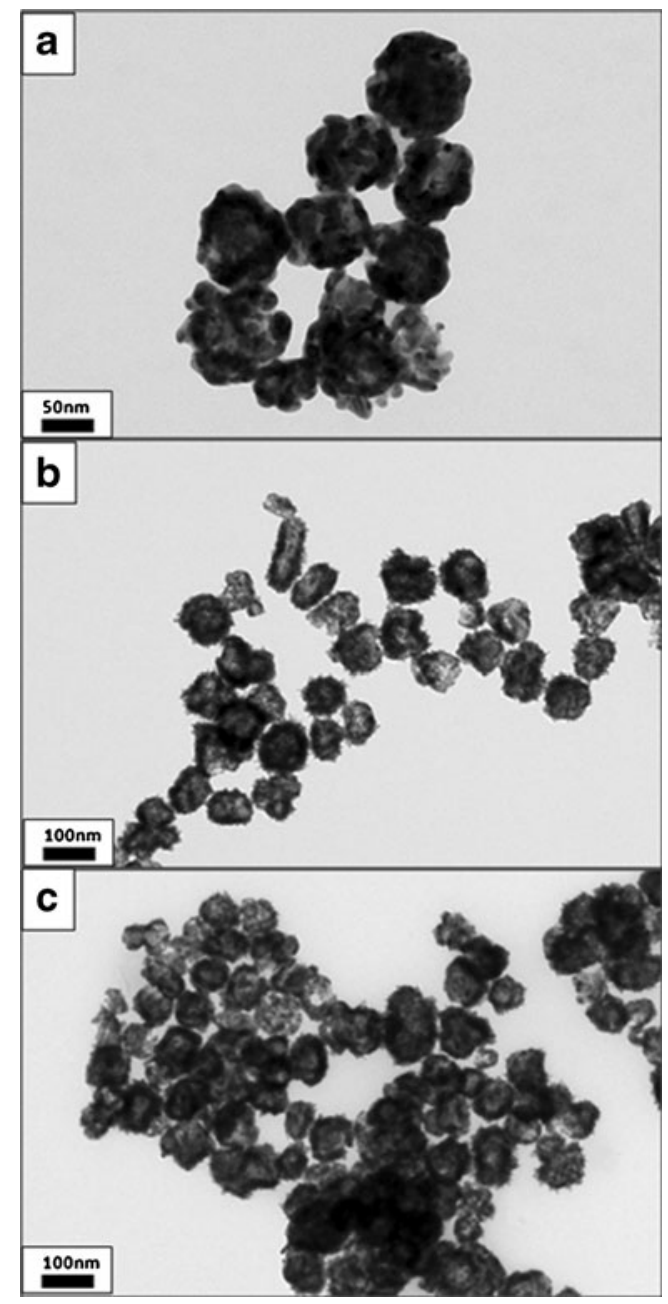

Fig. 5 TEM images of the hollow urchin-like AuNPs at different intervals. a $10 \mathrm{~s} ;$ b $30 \mathrm{~s}$; c $1 \mathrm{~min}$ 


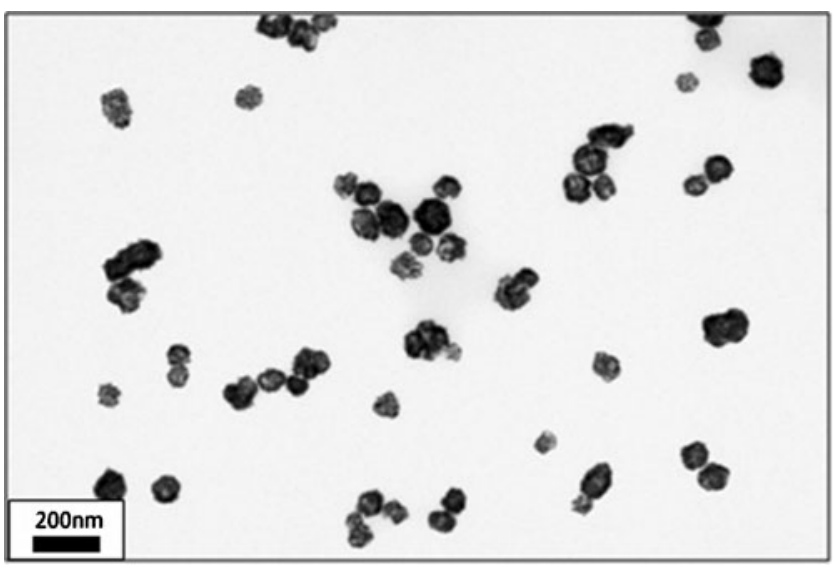

Fig. 6 TEM image of the hollow urchin-like AuNPs via adding $\mathrm{HAuCl}_{4}$ to concentrated AgNPs

the urchin-like structure (Fig. 3d). Temporal evolution of the hollow urchin-like structure was studied and it showed that the formation of these unique structured NPs is a fast process. Upon addition of all the ingredients, the galvanic replacement reaction occurs in $10 \mathrm{~s}$, resulting hollow-structured NPs with rough surface. After $30 \mathrm{~s}$, the tips grow around the hollow NPs via the reduction of $\mathrm{HAuCl}_{4}$ by ascorbic acid. No apparent difference is observed in the later incubation time except a structure optimization from the diffusion process and Oswald ripening (Fig. 5). Attention should be paid that the feeding order of the reactants slightly makes effects on the formation of the hollow urchin-like AuNPs. If $\mathrm{HAuCl}_{4}$ is added to the concentrated Ag NPs, similar structure can be obtained; however, the NPs show less uniform distribution (Fig. 6), which may originate from the uneven galvanic replacement reaction between the seeds and $\mathrm{HAuCl}_{4}$ because of the limited $\mathrm{HAuCl}_{4}$ available at the initial stage.

The morphology of the AgNP seeds controls the shape and size of the resulting AuNPs. Changing the AgNP size can effectively tune the diameter of the corresponding gold structures. After centrifugation at lower speed, the supernatant of the Ag colloid is further centrifugated at higher speed, thereby AgNPs with smaller size were roughly obtained (inset in Fig. 7a).

Using these AgNP seeds $\left(d_{\mathrm{av}}=46 \mathrm{~nm}\right)$, we synthesized hollow urchin-like AuNPs with average diameter of $95 \pm 15 \mathrm{~nm}$

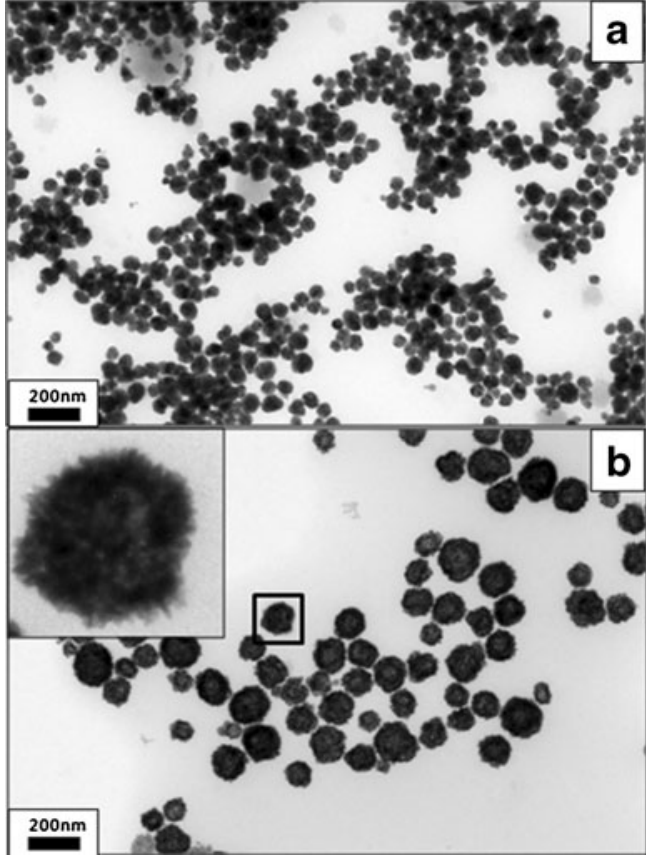

Fig. 8 TEM images of a AgNPs obtained from (ethylene) glycol system and $\mathbf{b}$ hollow urchin-like AuNPs via using the above AgNPs as seeds. Inset: A larger view for the AuNPs in b

(Fig. 7a). Their SPR peak can thus be regulated to $800 \mathrm{~nm}$ owing to the morphology change (Fig. 7b), and the shoulder peak at around $560 \mathrm{~nm}$ (also observable in curve 3, Fig. 2) is related to the gold tips, which is always detectable in previous reports [33,34]. Additionally, in Figs. If and 7a, some fiber-like structured particles are also observable, which should originate from the similar morphological AgNPs (inset in Fig. 7a). To exclude the presence of $\mathrm{Ag}$ nanofibers and the broad distribution of the AgNPs, we applied AgNPs obtained from EG system as seeds for the formation of hollow urchin-like NPs. As expected, similar structures were generated except for a more compact tips configuration (Fig. 8), which may result from the different surface properties of the Ag seeds.

It is anticipated that such structured AuNPs behave excellent catalytic performance. We selected the reduction of 4-NP to 4-aminophenol by $\mathrm{NaBH}_{4}$ in aqueous media as a model reaction. The catalytic properties of the hollow
Fig. 7 a SEM image and $\mathbf{b}$ UV-vis spectrum of hollow urchin-like AuNPs obtained by using smaller AgNPs as seeds. Inset: TEM image of AgNPs with smaller average diameter
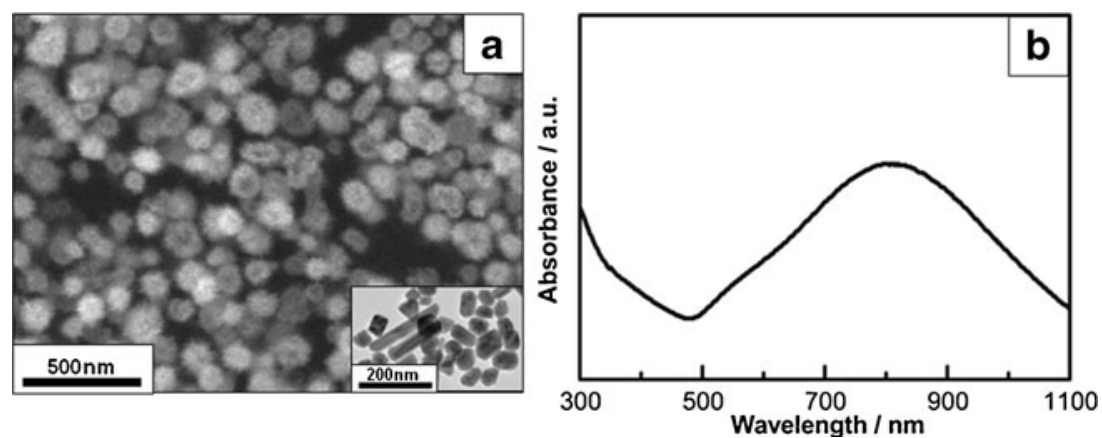
Fig. 9 Time-dependent evolution of UV-vis spectra for the reduction of 4-NP by $\mathrm{NaBH}_{4}$ using a hollow urchinlike and b spherical AuNPs as catalysts. Inset: The relationship between $-\ln \left(C_{\mathrm{t}} / C_{0}\right)$ versus time $(t)$ in a hollow urchin-like and $\mathbf{b}$ spherical AuNPs system
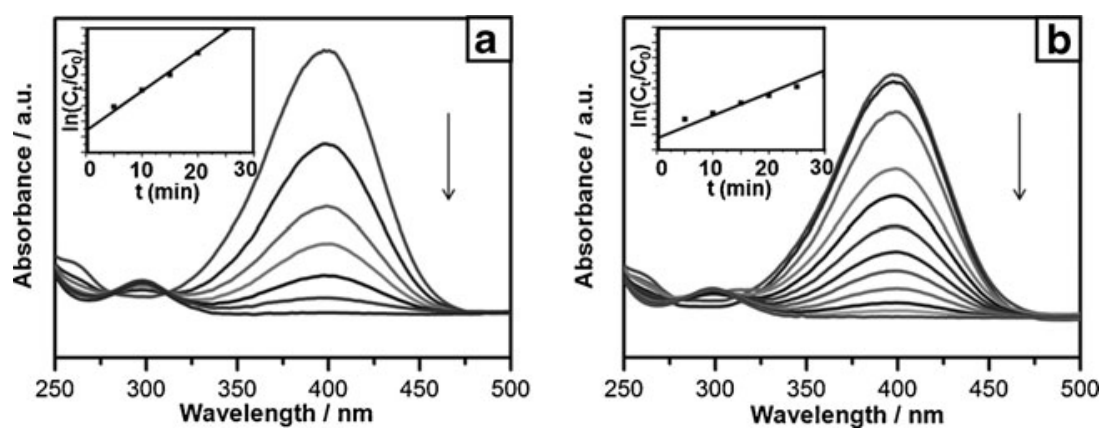

urchin-like AuNPs and spherical AuNPs synthesized in the similar system without AgNPs as seeds were compared based on their respective degrade effects. When hollow urchin-like AuNPs were used as catalysts, the complete degradation was detected in $30 \mathrm{~min}$; however, using equivalent spherical AuNPs as catalysts accomplished the degradation in 55 min (Fig. 9a, b). Furthermore, in Fig. 9b, the first two curves nearly overlap, indicating that a longer period of time is required for the 4-NP to adsorb onto the surface of the spherical AuNPs due to their lower activity [24].

Considering this reaction a first-order reaction, the rate constant is determined by the slope of the linear fit of $-\mathrm{ln}$ $\left(C_{\mathrm{t}} / C_{0}\right)$ versus time, where $C_{\mathrm{t}} / C_{0}$ represents the ratio of 4NP concentration at time $t$ and 0 as calculated based on their corresponding absorbance intensity in the kinetic UV-vis spectra. The rate constants are 0.124 and $0.073 \mathrm{~min}^{-1}$ for the reaction by using hollow urchin-like and spherical AuNPs as catalysts, respectively (insets in Fig. 9a, b). Following the previous discussion on the catalyst, a relatively large surface permits the oxidation and reduction reaction occurring together while a small particle size is essential to keep its high activity [24]. The hollow urchin-like AuNPs fit this point well. The sharp tips and thin shell not only render them high activity, but keep linked to avoid separating the two half

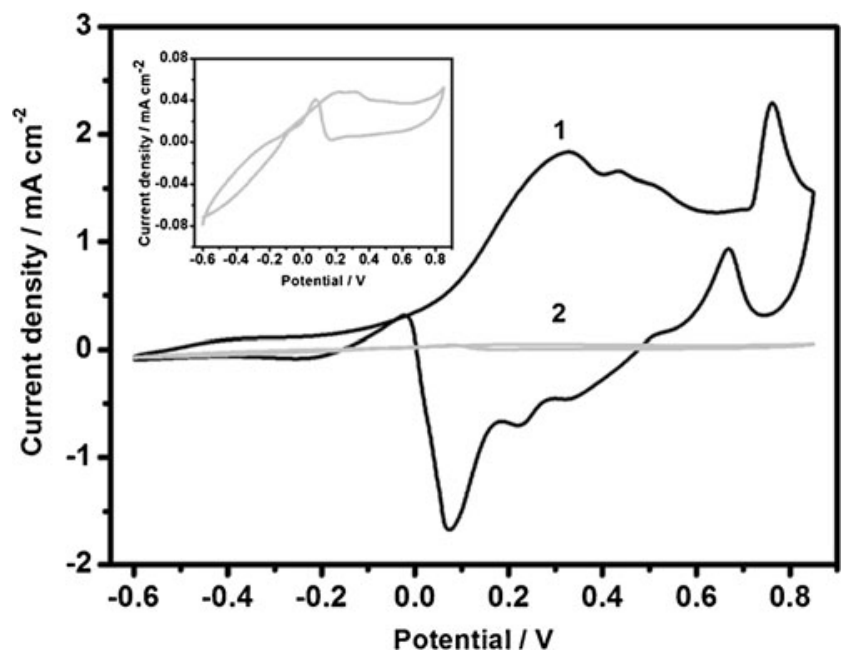

Fig. $10 \mathrm{CVs}$ for the oxidation of glucose $(1.33 \mathrm{mM})$ at hollow urchinlike (curve 1) and spherical (curve 2) AuNPs electrodes in $0.1 \mathrm{M}$ $\mathrm{NaOH}$ at $50 \mathrm{mV} \mathrm{s}^{-1}$. Inset: A magnified view for curve 2 reactions. Therefore, a higher catalytic activity was achieved for the hollow urchin-like AuNPs.

The catalytic property of the hollow urchin-like AuNPs was further evaluated by exploiting the NPs in the electrocatalytic oxidation of glucose. The CV curves of the glucose oxidation using gold as catalyst are documented in previous reports [33, 35, 36]. Typically, using the spherical AuNPs electrode (Fig. 10), an oxidation potential located at $0.21 \mathrm{~V}$ is corresponding to the electrosorption of glucose to form adsorbed intermediate, which releases one proton per glucose molecule. These intermediates accumulated and occupied on the active sites of the electrode surface, inhibiting the direct oxidation of glucose. As a more positive potential is attained $(0.48 \mathrm{~V})$, the intermediates are catalytically oxidized by the formed adsorbed $\mathrm{OH}$, leaving free $\mathrm{Au}$ active sites to directly oxidize the glucose. However, the formation of the gold oxide at a higher potential blocks the glucose to adsorb onto the active sites. As the negative potential is carrying on, the peak at $-0.07 \mathrm{~V}$ is observed, relating to the formation of intermediates on the electrode surface again. Compared to the result by using spherical AuNPs, a much higher current density is achieved for the direct oxidation of glucose around $0.32 \mathrm{~V}$ for the hollow urchin-like gold system, indicating a much faster electron transfer on the electrode surface and thus higher catalytic performance [37]. During the negative potential sweep, glucose can be oxidized again when the Au surface is free from oxides, leading to the cathodic reoxidation peak at $0.66 \mathrm{~V}$. This peak along with the peak located at $0.76 \mathrm{~V}$ are rarely detected for the spherical AuNPs, which probably results from the special hollow urchin-like structure and needs further detailed investigation.

In summary, we successfully synthesized hollow urchinlike gold nanoparticles by using silver nanoparticles as sacrificial seeds followed by reduction of excessive $\mathrm{HAuCl}_{4}$ with ascorbic acid. This unique structure makes them excellent application in optics and catalysis fields. Similar strategies will be adopted for generation of other hollow urchinlike metal nanoparticles to dig their wider application.

Acknowledgments This work was supported by National Natural Science Foundation of China (grant no. 21103018) and Jilin Provincial Science and Technology Development Foundation (grant no. 201101010). 
Open Access This article is distributed under the terms of the Creative Commons Attribution License which permits any use, distribution and reproduction in any medium, provided the original author(s) and source are credited.

\section{References}

1. Daniel M-C, Astruc D (2003) Gold nanoparticles: assembly, supramolecular chemistry, quantum-size-related properties, and applications toward biology, catalysis, and nanotechnology. Chem Rev 104:293-346. doi:10.1021/cr030698+

2. Hu M, Chen J, Li Z-Y, Au L, Hartland GV, Li X, Marquez M, Xia $Y$ (2006) Gold nanostructures: engineering their plasmonic properties for biomedical applications. Chem Soc Rev 35:1084-1094. doi:10.1039/b517615h

3. Yam VW-W, Cheng EC-C (2008) Highlights on the recent advances in gold chemistry-a photophysical perspective. Chem Soc Rev 37:1806-1813. doi:10.1039/b708615f

4. Soulé J-F, Miyamura H, Kobayashi S (2011) Powerful amide synthesis from alcohols and amines under aerobic conditions catalyzed by gold or gold/iron, -nickel or -cobalt nanoparticles. J Am Chem Soc 133:18550-18553. doi:10.1021/ja2080086

5. Zhang Y, Cui X, Shi F, Deng Y (2011) Nano-gold catalysis in fine chemical synthesis. Chem Rev. doi:10.1021/cr200260m

6. Haruta M (2004) Gold as a novel catalyst in the 21st century: preparation, working mechanism and applications. Gold Bull 37:27-36. doi:10.1007/BF03215514

7. Nehl CL, Hafner JH (2008) Shape-dependent plasmon resonances of gold nanoparticles. J Mater Chem 18:2415-2419. doi:10.1039/ b714950f

8. You H, Ji Y, Wang L, Yang S, Yang Z, Fang J, Song X, Ding B (2012) Interface synthesis of gold mesocrystals with highly roughened surfaces for surface-enhanced Raman spectroscopy. J Mater Chem. doi:10.1039/c1jm13211c

9. Sperling RA, Rivera Gil P, Zhang F, Zanella M, Parak WJ (2008) Biological applications of gold nanoparticles. Chem Soc Rev 37:1896-1908. doi:10.1039/b712170a

10. Lu L, Ai K, Ozaki Y (2008) Environmentally friendly synthesis of highly monodisperse biocompatible gold nanoparticles with urchin-like shape. Langmuir 24:1058-1063. doi:10.1021/ la702886q

11. Lim B, Xia Y (2011) Metal nanocrystals with highly branched morphologies. Angew Chem In Ed 50:76-85. doi:10.1002/ anie. 201002024

12. Li J, Wu J, Zhang X, Liu Y, Zhou D, Sun H, Zhang H, Yang B (2011) Controllable synthesis of stable urchin-like gold nanoparticles using hydroquinone to tune the reactivity of gold chloride. J Phys Chem C 115:3630-3637. doi:10.1021/ jp1119074

13. Broek BVd, Frederix F, Bonroy K, Jans H, Jans K, Borghs G, Maes G (2011) Shape-controlled synthesis of NIR absorbing branched gold nanoparticles and morphology stabilization with alkanethiols. Nanotechnology 22:015601. doi:10.1088/09574484/22/1/015601

14. Waqqar A, Kooij ES, Arend van S, Bene P (2010) Controlling the morphology of multi-branched gold nanoparticles. Nanotechnology 21:125605. doi:10.1088/0957-4484/21/12/125605

15. Bakr OM, Wunsch BH, Stellacci F (2006) High-yield synthesis of multi-branched urchin-like gold nanoparticles. Chem Mater 18:3297-3301. doi:10.1021/cm060681i

16. Kuo C-H, Huang MH (2005) Synthesis of branched gold nanocrystals by a seeding growth approach. Langmuir 21:2012-2016. doi:10.1021/la0476332
17. Sun Y, Mayers B, Xia Y (2003) Metal nanostructures with hollow interiors. Adv Mater 15:641-646. doi:10.1002/adma.200301639

18. Hu J, Chen M, Fang X, Wu L (2011) Fabrication and application of inorganic hollow spheres. Chem Soc Rev 40:5472-5491. doi:10.1039/c1cs15103g

19. Liang H-P, Wan L-J, Bai C-L, Jiang L (2005) Gold hollow nanospheres: tunable surface plasmon resonance controlled by interiorcavity sizes. J Phys Chem B 109:7795-7800. doi:10.1021/ jp045006f

20. Liu J, Liu F, Gao K, Wu J, Xue D (2009) Recent developments in the chemical synthesis of inorganic porous capsules. J Mater Chem 19:6073-6084. doi:10.1039/b900116f

21. Ortiz N, Skrabalak SE (2011) Controlling the growth kinetics of nanocrystals via galvanic replacement: synthesis of au tetrapods and star-shaped decahedra. Crys Growth Des 11:3545-3550. doi: $10.1021 / \mathrm{cg} 200484 \mathrm{~m}$

22. Xia Y, Li W, Cobley CM, Chen J, Xia X, Zhang Q, Yang M, Cho EC, Brown PK (2011) Gold nanocages: from synthesis to theranostic applications. Acc Chem Res 44:914-924. doi:10.1021/ ar200061q

23. Chen J, Yang M, Zhang Q, Cho EC, Cobley CM, Kim C, Glaus C, Wang LV, Welch MJ, Xia Y (2010) Gold nanocages: a novel class of multifunctional nanomaterials for theranostic applications. Adv Funct Mater 20:3684-3694. doi:10.1002/ adfm.201001329

24. Zeng J, Zhang Q, Chen J, Xia Y (2010) A comparison study of the catalytic properties of Au-based nanocages, nanoboxes, and nanoparticles. Nano Lett 10:30-35. doi:10.1021/n1903062e

25. Personick ML, Langille MR, Zhang J, Mirkin CA (2011) Shape control of gold nanoparticles by silver underpotential deposition. Nano Lett 11:3394-3398. doi:10.1021/n1201796s

26. Skrabalak SE, Chen J, Sun Y, Lu X, Au L, Cobley CM, Xia Y (2008) Gold nanocages: synthesis, properties, and applications. Acc Chem Res 41:1587-1595. doi:10.1021/ar800018v

27. Sun Y, Xia Y (2004) Mechanistic study on the replacement reaction between silver nanostructures and chloroauric acid in aqueous medium. J Am Chem Soc 126:3892-3901. doi: $10.1021 / \mathrm{ja} 039734 \mathrm{c}$

28. Lu X, Tuan HY, Chen J, Li ZY, Korgel BA, Xia Y (2007) Mechanistic studies on the galvanic replacement reaction between multiply twinned particles of $\mathrm{Ag}$ and $\mathrm{HAuCl}_{4}$ in an organic medium. J Am Chem Soc 129:1733-1742. doi:10.1021/ja067800f

29. Ataee-Esfahani H, Nemoto Y, Wang L, Yamauchi Y (2011) Rational synthesis of Pt spheres with hollow interior and nanosponge shell using silica particles as template. Chem Commun 47:3885-3887. doi:10.1039/c0cc05233g

30. Sun Y, Wiley B, Li Z-Y, Xia Y (2004) Synthesis and optical properties of nanorattles and multiple-walled nanoshells/nanotubes made of metal alloys. J Am Chem Soc 126:9399-9406. doi: $10.1021 / \mathrm{ja} 048789 \mathrm{r}$

31. Gooding JJ, Praig VG, Hall EAH (1998) Platinum-catalyzed enzyme electrodes immobilized on gold using self-assembled layers. Anal Chem 70:2396-2402. doi:10.1021/ac971035t

32. Liu Y, Hight Walker AR (2011) Preferential outward diffusion of $\mathrm{Cu}$ during unconventional galvanic replacement reactions between $\mathrm{HAuCl}_{4}$ and surface-limited $\mathrm{Cu}$ nanocrystals. ACS Nano 5:68436854. doi:10.1021/nn200565y

33. Xu F, Cui K, Sun Y, Guo C, Liu Z, Zhang Y, Shi Y, Li Z (2010) Facile synthesis of urchin-like gold submicrostructures for nonenzymatic glucose sensing. Talanta 82:1845-1852. doi:10.1016/ j.talanta.2010.07.087

34. Pandian Senthil K, Isabel P-S, Benito R-G, Abajo FJGd, Luis MLM (2008) High-yield synthesis and optical response of gold nanostars. Nanotechnology 19:015606. doi:10.1088/0957-4484/19/01/ 015606 
35. Zhang H, Xu J-J, Chen H-Y (2008) Shape-controlled gold nanoarchitectures: synthesis, superhydrophobicity, and electrocatalytic properties. J Phys Chem C 112:13886-13892. doi:10.1021/ jp802012h

36. Li Y, Song Y-Y, Yang C, Xia X-H (2007) Hydrogen bubble dynamic template synthesis of porous gold for nonenzymatic electrochemical detection of glucose. Electrochem Commun 9:981-988. doi:10.1016/j.elecom.2006.11.035

37. Zhao J, Kong X, Shi W, Shao M, Han J, Wei M, Evans DG, Duan X (2011) Self-assembly of layered double hydroxide nanosheets/Au nanoparticles ultrathin films for enzyme-free electrocatalysis of glucose. J Mater Chem 21:13926-13933. doi:10.1039/c1jm12060c 